\title{
Evaluación de calcio y fósforo fino y grueso en la dieta de gallinas ponedoras en dos frecuencias de suministro sobre la calidad de la cáscara de huevo
}

\author{
Evaluation of calcium and phosphorus fine and coarse in the diet of laying hens in two frequencys \\ of feeding supply on the quality of egg shell
}

Pedro Ciriaco C. ${ }^{1}$; Víctor Escalante A.²

\begin{abstract}
Resumen
Se evaluó el efecto de dos tamaños de partículas de calcio y fósforo, fino y grueso, suministrado a gallinas ponedoras, a través de la dieta, en una y dos frecuencias por día, evaluándose espesor de la cáscara, porcentaje de postura, masa de huevo, consumo de alimento, peso promedio del huevo, conversión alimenticia y mérito económico. Se distribuyeron 384 gallinas de 36 semanas de edad, en 8 tratamientos, con tres repeticiones, utilizándose el diseño completamente al azar, con un arreglo factorial de $2 \times 2 \times 2$ (dos tamaños de partícula de calcio, dos tamaños de partícula del fósforo, dos frecuencias de suministro del alimento). Hubo diferencias estadísticas para el espesor de la cáscara, donde las gallinas que consumieron calcio y fósforo grueso tuvieron los mayores espesores de cáscara. No hubo diferencias significativas para el porcentaje de postura y el peso promedio del huevo. Las aves que consumieron calcio grueso tuvieron las menores masas de huevo. Las gallinas que fueron alimentadas con calcio grueso y en dos frecuencias de suministro tuvieron el mayor consumo de alimento. El tratamiento con calcio fino, fosforo grueso y una sola frecuencia de suministro tuvo la mejor conversión alimenticia. El calcio grueso ha tenido mayor tiempo de permanencia en la circulación sanguínea y ha estado disponible cuando se realiza la calcificación de la cáscara del huevo. Asimismo, el fósforo grueso ha ayudado en el traslado de calcio de los huesos hacia la cáscara, en el momento de mayor necesidad de calcio.
\end{abstract}

Palabras clave: calcio; fósforo fino y grueso; espesor de cáscara.

\begin{abstract}
We evaluated the effect of two particle sizes of calcium and phosphorus, fine and coarse, given to laying hens through their diets, in one and two daily frequencies; evaluating egg shell thickness, egg laying percentage, egg mass, food consumption, egg weight average, feed conversion and economic profit. A total of 384 laying hens of 36 weeks of age were distributed in 8 treatments, with three replicates, using a completely randomized design, with a factorial arrangement of $2 \times 2 \times 2$ (two calcium particle sizes, two phosphorus particle sizes, two food supply frequencies). Statistical differences were found for egg shell thickness, where hens that consumed coarse calcium and phosphorus had the highest egg shell thickness. There were no significant differences for egg laying percentage and egg weight. Hens that consumed coarse calcium had the lowest egg mass. Those that were fed with coarse calcium and in two supply frequencies had the highest food intake. The treatment with fine calcium, coarse phosphorus and a single frequency of supply had the best feed conversion. Coarse calcium had a longer permanence period in the bloodstream and was available at the time of egg shell calcification. Also, coarse phosphorus has aided in calcium displacement from the bones to the egg shells at the time of highest calcium demand.
\end{abstract}

Keywords: Calcium; fine and coarse phosphorus; egg shell thickness.

1. Departamento de Producción Animal, Facultad de Zootecnia, Universidad Nacional Agraria La Molina, Lima (Perú).

2. Ingeniero Zootecnista, práctica privada. Email: pciriaco@lamolina.edu.pe 


\section{Introducción}

Las gallinas, para calcificar la cáscara del huevo, utilizan el calcio proveniente del alimento y de su propia médula ósea, proceso que se realiza por las noches; cuando no se encuentra suficiente calcio disponible para calcificar los huevos se ocasionan cáscaras de menor espesor o defectuosas (rotas, rajadas, arrugadas); asimismo, utilizan más fósforo para la resorción de calcio de los huesos (De Blas y Gonzales, 1991). Utilizando calcio y fósforo de partículas gruesas se podría evitar huevos frágiles, debido a que se reduce la velocidad de disolución en el tracto digestivo y retrasa su absorción total. Otra forma, sería la de suministrar el alimento diario dividido en dos partes, una en la mañana y otra en la tarde, tratando de mantener los minerales mas constantes en el torrente sanguíneo.

La presentación del carbonato de calcio $\left(\mathrm{CaCO}_{3}\right)$ y fosfato dicálcico es en forma de harina, existiendo la forma granulada de $2 \mathrm{~mm}$ de espesor para el $\mathrm{CaCO}_{3}$ y de $1.5 \mathrm{~mm}$ para el fosfato dicálcico (Scott, M., Neishem, M. y Young, R., 1991; Keshavarz, 1994; Rao, S., Roland, A. y Gordon, R., 1995).

La calidad de la cáscara del huevo es afectada negativamente cuando se utiliza el $\mathrm{CaCO}_{3}$ en forma de harina, observándose poca retención en el organismo (Cheng y Coon, 1990; Frost, T., Roland, A. y Untawale, G., 1990). Sin embargo, Guinotte y Nys (1991), reportaron una mejor calidad de la cáscara del huevo en aves alimentadas con dietas donde se incluía una fuente de calcio en forma granulada. Sauveur (1993), observó que la utilización de calcio en forma granulada mejora el espesor de la cáscara, cuando las gallinas son criadas a una temperatura superior a $\operatorname{los} 30^{\circ} \mathrm{C}$ y cuando son adultas (más de 56 semanas de edad). Keshavarz (1994) sugiere que debe usarse un $30 \%$ a $50 \%$ de carbonato de calcio en forma granulada para mejorar su retención en la molleja. Asimismo, Bingfan y Coon (1997) observaron una baja retención en la molleja cuando se utilizó carbonato de calcio de partículas pequeñas. Tobin (2004) trabajó con dietas conteniendo carbonato de calcio fino $(75 \%)$ y granulado $(25 \%)$, no encontrando diferencias significativas en el consumo de alimento, peso corporal, producción de huevos, peso del huevo y el espesor de la cáscara del huevo. Sin embargo, Rostagno, H., Albino, J., Doncele, P. y Oliveira, R. (2005), encontraron que la mezcla de un $75 \%$ de calcio en forma de harina y un $25 \%$ de calcio granulado mejora la calidad de la cáscara. Cuca, M., Valdes, V., Pro, A., Suarez, M. y Figueroa, J. (2007), trabajaron con $50 \%$ de granulado encontrando una mejor calidad de cáscara. Por otro lado, Saunders, J., Maclsaac, J., Kover, D. y Anderson, D. (2009) observaron que a medida que se incrementaba el diámetro de la partícula del carbonato de calcio, mejoraba el espesor de la cascara, pero el porcentaje de postura y el consumo de alimento disminuían. Ccoicca (2009), utilizó calcio de 1 a $1.2 \mathrm{~mm}$ de diámetro, no encontrando diferencias significativas para el espesor de la cascara; sin embargo, sí hubo diferencias para número de huevos, masa de huevos, peso promedio de huevo y porcentaje de postura a favor del carbonato de calcio fino.

Fuentes granuladas de fosforo tienen un mejor valor biológico comparado con aquellas que son de menores tamaños de partícula (Burnell, T., Cromwell, G. y Stahly, T., 1990; Keshavarz y Nakajima, 1993; Rao et al., 1995). Durante la calcificación de la cáscara del huevo, parte del calcio sale de los huesos haciendo que los iones de calcio y de fósforo entren en la sangre. Después los iones de fósforo son eliminados por los riñones. Para volver a llenar las reservas de fósforo se requiere alimentar con fósforo. Cuando hay una falta significativa de fósforo, la producción de huevo baja y la mortalidad aumenta (Williams, Ahmad y Balander, 2004).

El objetivo de la presente investigación es evaluar el efecto de dos tamaños de partículas fino y grueso de calcio $\mathrm{y}$ fósforo, suministrando la dieta en una o dos frecuencias por día, en gallinas Hy Line Brown y evaluando el espesor de la cáscara, porcentaje de postura, peso promedio del huevo, masa de huevo, consumo de alimento, conversión alimenticia y retribución económica del alimento.

\section{Materiales y métodos}

El estudio se llevó a cabo en las instalaciones de la Unidad Experimental de Avicultura de la Universidad Nacional Agraria La Molina, durante 12 semanas. Se utilizaron 384 gallinas de postura de la línea Hy Line Brown de 36 semanas de edad, con pesos homogéneos y óptimo estado de salud. Las aves fueron alojadas en 96 jaulas de alambre galvanizado con dimensiones de 60 cm largo x $40 \mathrm{~cm}$ de alto x $40 \mathrm{~cm}$ de ancho, alojándose 4 gallinas por jaula, que estuvieron dentro de un galpón tipo "abierto", realizándose el manejo de cortinas de acuerdo a la presentación de variaciones de temperaturas con el fin de brindar el grado adecuado de ventilación y evitar la presentación de estrés. Se aplicaron correctamente todas las medidas de bioseguridad.

El alimento fue formulado de acuerdo con los requerimientos nutricionales para gallinas ponedoras (Hy Line, 2011). La composición porcentual y valor calculado del aporte nutritivo de las dietas se encuentra en la Tabla 1. Se utilizó como fuente de calcio el carbonato de calcio fino de $0.4 \mathrm{~mm}$ y grueso de $2.5 \mathrm{~mm}$ de diámetro, con contenido de calcio de $38 \%$; como fuente de fósforo el fosfato dicálcico fino de $0.5 \mathrm{~mm}$ y grueso de $1.5 \mathrm{~mm}$ de diámetro que contiene $18 \%$ de fosforo disponible y $21 \%$ de calcio. El suministro diario del alimento fue de 130 gr/ave/día, para poder medir el consumo real, que podría estar influido por el consumo de los minerales granulados.

El diseño estadístico empleado fue el completamente al

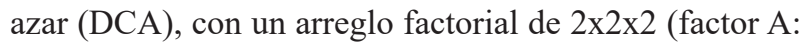
dos tamaños de carbonato de calcio; factor B: dos tamaños de fosfato dicálcico; factor C: dos periodos de suministro de alimento), haciendo un total de 8 tratamientos. Se emplearon 3 repeticiones por tratamiento, con 24 aves 
cada una. La comparación de medias de los tratamientos fue con la prueba de Duncan con $\alpha$ : $5 \%$; siendo los datos en porcentaje transformados con la fórmula: Arc Sen para que se ajusten a la curva normal (Calzada, 1982).

La evaluación de los parámetros fue cada semana. La colección de huevos se realizó diariamente en un mismo horario registrándose todos los datos concernientes a la producción.

Para el espesor de la cáscara, se obtuvieron huevos al azar, midiéndose con un micro calibrador la parte del ecuador y los polos, tomándose el promedio aritmético de estos valores. El alimento se suministró en los horarios de 8:00 am y 2:00 pm de acuerdo con los tratamientos evaluados de una o dos frecuencias por día. Los parámetros evaluados fueron: Espesor de la cáscara, porcentaje de postura, peso promedio de huevo, masa de huevo, consumo de alimento, conversión alimenticia y retribución económica del alimento.

\section{Resultados y discusión}

Los resultados se muestran en la Tabla 2. Para el espesor de la cáscara, existen diferencias significativas; las aves alimentadas con la dieta donde se incluyeron calcio y fósforo grueso proporcionaron el mayor espesor y por

Tabla 1. Composición porcentual de las dietas y el valor nutritivo estimado

\begin{tabular}{|c|c|c|c|c|}
\hline \multirow{2}{*}{ INSUMOS } & \multicolumn{4}{|c|}{ DIETAS (\%) } \\
\hline & 1 & 2 & 3 & 4 \\
\hline Maíz amarillo duro & 56.00 & 56.00 & 56.00 & 56.00 \\
\hline Torta de soya, $46 \%$ & 19.66 & 19.66 & 19.66 & 19.66 \\
\hline Subproducto de trigo & 5.00 & 5.00 & 5.00 & 5.00 \\
\hline Harina de pescado prime, 65 & 6.00 & 6.00 & 6.00 & 6.00 \\
\hline Aceite de palma & 1.28 & 1.28 & 1.28 & 1.28 \\
\hline Carbonato de calcio fino & 9.00 & 9.00 & 0.00 & 0.00 \\
\hline Carbonato de calcio grueso & 0.00 & 0.00 & 9.00 & 9.00 \\
\hline Fosfato dicálcico grueso & 0.00 & 2.60 & 0.00 & 2.60 \\
\hline Fosfato dicálcico fino & 2.60 & 0.00 & 2.60 & 0.00 \\
\hline Premezcla de postura & 0.10 & 0.10 & 0.10 & 0.10 \\
\hline Sal común & 0.10 & 0.10 & 0.10 & 0.10 \\
\hline DL- Metionina & 0.13 & 0.13 & 0.13 & 0.13 \\
\hline Cloruro de colina, $60 \%$ & 0.07 & 0.07 & 0.07 & 0.07 \\
\hline Zinc Bacitracina & 0.06 & 0.06 & 0.06 & 0.06 \\
\hline EM (Mcal/Kg.) & \multicolumn{4}{|c|}{2.78} \\
\hline Proteína total & \multicolumn{4}{|c|}{16.00} \\
\hline Metionina Total & \multicolumn{4}{|c|}{0.40} \\
\hline Lisina total & \multicolumn{4}{|c|}{0.92} \\
\hline Metionina + Cistina total & \multicolumn{4}{|c|}{0.81} \\
\hline Treonina total & \multicolumn{4}{|c|}{0.63} \\
\hline Triptófano total & \multicolumn{4}{|c|}{0.19} \\
\hline Calcio & \multicolumn{4}{|c|}{4.00} \\
\hline Fosforo disponible & \multicolumn{4}{|c|}{0.36} \\
\hline Sodio & \multicolumn{4}{|c|}{0.16} \\
\hline Ácido linoleico & \multicolumn{4}{|c|}{0.91} \\
\hline
\end{tabular}

$1=$ dieta con calcio fino y fósforo fino, $2=$ dieta con calcio fino y fósforo grueso, $3=$ dieta con calcio grueso y fósforo fino, $4=$ dieta con calcio grueso y fósforo grueso. Los tratamientos fueron:

T-1: Calcio fino y fósforo fino; suministrado una vez por día.

T-2: Calcio fino y fósforo fino; suministrado dos veces por día.

T-3: Calcio fino y fósforo grueso; suministrado una vez por día.

T-4: Calcio fino y fósforo grueso; suministrado dos veces por día.

T-5: Calcio grueso y fósforo fino; suministrado una vez por día.

T-6: Calcio grueso y fósforo fino; suministrado dos veces por día.

T-7: Calcio grueso y fósforo grueso; suministrado una vez por día.

T-8: Calcio grueso y fósforo grueso; suministrado dos veces por día. 
lo tanto una mejor calidad de la cáscara del huevo, sin embargo no hubo diferencias entre la interacción de ambos minerales con las frecuencias de suministro de alimento.

La utilización de calcio y fósforo grueso incluidos en la ración produjeron mayor valor del espesor de la cáscara, indicando que hay una mayor retención de calcio y fósforo en la molleja de la gallina ponedora, así como una lenta liberación del mismo, que permite una mayor permanencia de los minerales circulantes en la sangre y por lo tanto una mayor utilización de calcio y fósforo. Asimismo, el uso de fosfato dicálcico grueso también ha influido para el traslado de calcio hacia la cáscara, en el momento de mayor necesidad de este mineral para la calcificación de la cáscara del huevo, concordando con Sauveur (1993), Guinotte y Nyls (1991), Keshavarz (1994) y Bingfan y Coon (1997), quienes observaron una mayor solidez de la cáscara del huevo cuando utilizaron dietas donde se incluía calcio de partícula gruesa. Sin embargo, no concuerdan con Tobin (2004) y CCoicca (2009), quienes no hallaron diferencias en los valores del espesor de la cáscara del huevo cuando utilizaron dietas donde se incluyó calcio fino y grueso, probablemente por haber utilizado calcio grueso de menor diámetro al utilizado en el presente experimento.

No se encontraron diferencias significativas para el porcentaje de postura; la utilización de calcio y fosforo fino y grueso, la interacción de ambos y la interacción con la frecuencia de suministro de alimento, no afectaron el porcentaje de postura. Los resultados concuerdan

Tabla 2. Efecto del tamaño de partícula del calcio y fósforo y la frecuencia de suministro de alimento sobre la respuesta productiva de gallinas (36-48sem.)

\begin{tabular}{|c|c|c|c|c|c|c|c|}
\hline Tratamiento & & $\begin{array}{c}\text { Espesor } \\
\text { Cáscara } \\
(\mathrm{mm})\end{array}$ & $\begin{array}{c}\% \\
\text { Postura }\end{array}$ & $\begin{array}{c}\text { Peso } \\
\text { Huevo } \\
\text { (g) }\end{array}$ & $\begin{array}{c}\text { Masa } \\
\text { Huevo } \\
(\mathrm{Kg})\end{array}$ & $\begin{array}{c}\text { Consumo } \\
\text { Alimento } \\
\text { (g/ave/ } \\
\text { día) }\end{array}$ & $\begin{array}{l}\text { Conversión } \\
\text { Alimenticia }\end{array}$ \\
\hline 1 & & $0.3568^{\mathrm{c}}$ & $93.63^{\mathrm{a}}$ & $63.24^{\mathrm{a}}$ & $119.36^{\mathrm{a}}$ & $104.54^{\mathrm{dc}}$ & $1.766^{\mathrm{bc}}$ \\
\hline 2 & & $0.3508^{c}$ & $93.65^{\mathrm{a}}$ & $61.98^{\mathrm{a}}$ & $115.34^{\mathrm{ab}}$ & $109.42^{\mathrm{a}}$ & $1.913^{\mathrm{a}}$ \\
\hline 3 & & $0.3731^{\mathrm{b}}$ & $94.20^{\mathrm{a}}$ & $63.83^{\mathrm{a}}$ & $119.28^{\mathrm{a}}$ & $102.84^{\mathrm{d}}$ & $1.738 \mathrm{c}$ \\
\hline 4 & & $0.3791^{\mathrm{ab}}$ & $93.38^{\mathrm{a}}$ & $62.55^{\mathrm{a}}$ & $117.76^{\mathrm{ab}}$ & $108.53^{\mathrm{a}}$ & $1.858^{\mathrm{ab}}$ \\
\hline 5 & & $0.3785^{\mathrm{ab}}$ & $91.52^{\mathrm{a}}$ & $62.54^{\mathrm{a}}$ & $115.39^{\mathrm{ab}}$ & $106.03^{c}$ & $1.852^{\mathrm{abc}}$ \\
\hline 6 & & $0.3882^{\mathrm{ab}}$ & $90.68^{\mathrm{a}}$ & $62.43^{\mathrm{a}}$ & $114.11^{\mathrm{ab}}$ & $109.39^{\mathrm{a}}$ & $1.933^{\mathrm{a}}$ \\
\hline 7 & & $0.3912^{\mathrm{a}}$ & $88.98^{\mathrm{a}}$ & $62.68^{\mathrm{a}}$ & $112.44^{\mathrm{b}}$ & $106.47^{\mathrm{bc}}$ & $1.912^{\mathrm{a}}$ \\
\hline 8 & & $0.3902^{\mathrm{a}}$ & $91.17^{\mathrm{a}}$ & $62.00^{\mathrm{a}}$ & $113.95^{\mathrm{ab}}$ & $108.25^{\mathrm{ab}}$ & $1.916^{\mathrm{a}}$ \\
\hline Efecto Calcio & Fino & 0.3650 & 93.71 & 62.65 & 117.94 & 106.33 & 1.819 \\
\hline & Grueso & 0.3870 & 90.59 & 62.41 & 113.97 & 107.53 & 1.903 \\
\hline Efecto Fosforo & Fino & 0.3683 & 92.37 & 62.55 & 116.05 & 107.34 & 1.866 \\
\hline & Grueso & 0.3834 & 91.93 & 62.52 & 115.86 & 106.52 & 1.856 \\
\hline Efecto Frecuencia & Una vez & 0.3749 & 92.08 & 62.82 & 116.62 & 104.97 & 1.817 \\
\hline & Dos veces & 0.3770 & 92.22 & 62.24 & 115.29 & 108.89 & 1.905 \\
\hline \multicolumn{8}{|c|}{ Probabilidad } \\
\hline Calcio & & 0.0002 & 0.0295 & 0.6600 & 0.0136 & 0.0216 & 0.0085 \\
\hline Fósforo & & 0.0023 & 0.7209 & 0.9517 & 0.8822 & 0.0875 & 0.6925 \\
\hline Frecuencia & & 0.5384 & 0.9119 & 0.2971 & 0.3239 & 0.0001 & 0.0068 \\
\hline Calcio*Fosforo & & 0.0567 & 0.6354 & 0.8378 & 0.3096 & 0.2897 & 0.2393 \\
\hline Calcio*Frecuencia & & 0.5292 & 0.6628 & 0.7278 & 0.2855 & 0.0124 & 0.0975 \\
\hline \multirow[t]{2}{*}{ Fósforo* Frecuencia } & & 0.9226 & 0.6556 & 0.8487 & 0.3229 & 0.6584 & 0.3218 \\
\hline & & 0.1279 & 0.4368 & 0.4803 & 0.9539 & 0.1965 & 0.6289 \\
\hline
\end{tabular}

a, $\mathbf{b}, \mathbf{c}$, : Valores con letra diferente dentro de las columnas difieren significativamente.

$\mathrm{T} 1=$ Calcio fino y fósforo fino suministrado una vez al día, $\mathrm{T} 2=$ Calcio fino y fósforo fino suministrado dos veces al día, T3=Calcio fino y fósforo grueso suministrado una vez al día, T4= Calcio fino y fósforo grueso suministrado dos veces al día, T5= Calcio grueso y fósforo fino suministrado una vez al día, T6= Calcio grueso y fósforo fino suministrado dos veces al día, T7= Calcio grueso y fósforo grueso suministrado una vez al día, T8= Calcio grueso y fósforo grueso suministrado dos veces al día. 
con Tobin (2004) y Saunders et al. (2009), quienes no encontraron diferencias en sus valores comparativos cuando utilizaron carbonato de calcio fino y grueso. Sin embargo, no concuerdan con los hallazgos de CCoicca (2009), quien encontró resultados que mostraron diferencias significativas cuando se utilizó carbonato de calcio grueso, obteniendo los mejores valores cuando utilizó carbonato de calcio fino. Existe una tendencia a un menor porcentaje de postura cuando se utiliza calcio grueso, que se compara a lo reportado por Cuca (2007), quien observó una disminución en este parámetro a medida que se incrementaba el porcentaje de sustitución con calcio grueso, indicando que la utilización de calcio grueso puede influir en una menor densidad de nutrientes del alimento por el grosor que presenta al momento de la mezcla del alimento, conforme aumenta la sustitución con calcio grueso.

Para el peso promedio del huevo, no se encontraron diferencias estadísticas significativas; los minerales evaluados con dos formas de presentación o la interacción con la frecuencia de suministro no afectaron el peso del huevo. Los resultados concuerdan con Cuca (2007) y Saunders et al. (2009), pero no concuerda con CCoicca (2009), quien encontró un menor peso promedio del huevo al utilizar calcio grueso en la dieta de gallinas ponedoras.

Con respecto a la masa de huevos, se encontraron diferencias significativas para el efecto calcio grueso, donde los tratamientos presentan los menores valores de masa de huevo producida. Sin embargo, no fue afectada por la interacción con fosforo ni por la frecuencia de suministro del alimento. Estos resultados se deberían a una menor ingestión de nutrientes diferentes al calcio en las aves alimentadas con carbonato de calcio grueso, con respecto a las aves alimentadas con carbonato de calcio de partícula fina durante el periodo de calcificación de la cáscara de huevo, en los días de postura. Nutrientes como los aminoácidos (metionina y cistina) y la energía, tienen un efecto directo en la masa del huevo, lo cual pudo haber generado que las aves alimentadas con carbonato de calcio fino tengan una mayor masa de huevos y una tendencia a un mayor peso promedio de este que las aves alimentadas con carbonato de calcio de partícula gruesa, por haber escogido mayormente este mineral en momentos de mayor demanda, lo cual coincide con lo reportado por Keshavarz (1994), Cuca (2007) y Saunders et al. (2009).

En cuanto al consumo del alimento, se encontraron diferencias significativas para el efecto del calcio, frecuencia de suministro y la interacción de ambos, existiendo un mayor consumo de alimento. El mayor consumo se debió, probablemente, a la estimulación a la que eran sometidas cuando se les suministraba la segunda ración. Estos resultados no concuerdan con Bondi (1989), Guinotte, F. y Nys, Y. (1991), Sauveur (1993), Tobin (2004) y Saunders et al. (2009), quienes afirman que las aves alimentadas con calcio grueso presentan un menor consumo, debido al tamaño de partículas más grandes que permite ser diferenciado y escogido para su consumo en momentos de mayor necesidad de calcio y que provoca en el ave una sensación de llenura.

Se encontraron diferencias significativas para la conversión alimenticia, detectándose efectos del calcio y la frecuencia de suministro. Las aves alimentadas con calcio fino y fósforo grueso, con una sola frecuencia de suministro, obtuvieron la mejor respuesta; se reporta que el uso de calcio grueso incrementa la conversión alimenticia y que, más bien, no hay una mejora en la conversión alimenticia al realizar el suministro del alimento en dos frecuencias. Estos hallazgos concuerdan con Saunders et al. (2009), quien observó una menor eficiencia de conversión alimenticia en las gallinas

Tabla 3. Retribución económica por producción de huevo

\begin{tabular}{ccccccccc}
\hline & T1 & T2 & T3 & T4 & T5 & T6 & T7 & T8 \\
INGRESOS & & & & & & & & \\
Masa de huevos (kg) & 119.36 & 115.34 & 119.28 & 117.77 & 115.39 & 114.11 & 112.44 & 113.95 \\
Precio kg (S/.) & 6.00 & 6.00 & 6.00 & 6.00 & 6.00 & 6.00 & 6.00 & 6.00 \\
TOTAL (S/.) & 716.16 & 692.04 & 715.68 & 706.62 & 692.34 & 684.66 & 674.64 & 683.70 \\
EGRESOS & & & & & & & & \\
Consumo total (kg) & 210.76 & 220.60 & 207.32 & 218.79 & 213.75 & 220.53 & 214.65 & 218.24 \\
Costo kg. (S/.) & 1.60 & 1.60 & 1.65 & 1.65 & 1.61 & 1.61 & 1.67 & 1.67 \\
TOTAL (S/.) & 337.22 & 352.96 & 342.08 & 361.00 & 344.14 & 355.05 & 358.47 & 364.46 \\
\hline MERITO ECONOMICO & & & & & & & & \\
Por masa huevos (S/.) & 378.94 & 339.08 & 373.60 & 345.62 & 348.20 & 329.61 & 316.17 & 319.24 \\
Por kg de huevo (S/.) & 3.17 & 2.94 & 3.13 & 2.93 & 3.02 & 2.89 & 2.81 & 2.80 \\
\hline
\end{tabular}

T1= Calcio fino y fósforo fino suministrado una vez al día, T2= Calcio fino y fósforo fino suministrado dos veces al día, T3= Calcio fino y fósforo grueso suministrado una vez al día, T4 = Calcio fino fósforo grueso suministrado dos veces al día, T5= Calcio grueso y fósforo fino suministrado una vez al día, $\mathrm{T} 6=$ Calcio grueso y fósforo fino suministrado dos veces al día, $\mathrm{T} 7=$ Calcio grueso y fósforo grueso suministrado una vez al día, T8= Calcio grueso y fósforo grueso suministrado dos veces al día. 
ponedoras a medida que el calcio fino se remplazaba con el calcio grueso. En la Tabla 3, se muestra la retribución económica por tratamiento, basándose en los precios del huevo y los productos evaluados al mes de mayo del 2013.

La utilización de calcio y fosforo grueso en reemplazo de las partículas finas, disminuye la retribución económica por kg de huevo debido a que hay una tendencia a un menor porcentaje de postura cuando se utilizan estos minerales de partícula gruesa. Estos hallazgos concuerdan con Saunders et al. (2009), quienes observaron una disminución en el porcentaje de postura y masa de huevo cuando se reemplazaba calcio de partícula fina por gruesa y que la mejor relación donde no se afecta el rendimiento productivo es utilizar calcio fino y grueso en proporciones iguales.

\section{Conclusiones}

La utilización de carbonato de calcio y fosfato dicálcico de partículas gruesas en dietas de gallinas ponedoras, mejoraron la calidad de la cáscara del huevo. Sin embargo, disminuye la retribución económica por kilogramo de huevo. No obstante, se recomienda utilizar carbonato de calcio y fosfato dicálcico grueso para obtener mayor espesor de la cáscara del huevo; asimismo, realizar trabajos de investigación con estos minerales de partículas gruesas combinados en diferentes proporciones para hallar una mezcla donde no se afecte los parámetros productivos.

\section{Literatura citada}

Ahmad, H. y Balander, R. 2004. Physiological response of layers to alternative feeding regimen of calcium source and phosphorus level. International Journal of Poultry Science, 3(2): 100-111.

Bingfan, Z. y Coon, C. 1997. The relationship of calcium intake, source, size, solubility in vitro and in vivo, and gizzard limestone retention in laying hens. Poultry Science, 76: 1702-1706.

Burnell, T.; Cromwell, G. y Stahly, T. 1990. Effects of particle size on the biological availability of calcium and phosporus in defluorinated for chicks. Poultry Science, 69: 1110-1117.

Calzada, J. 1982. Métodos Estadísticos para la Investigación. (5 Edición). Lima, Perú: Ed. Agraria.

Cheng, T. y Coon, C. 1990. Effect of calcium source, particle size, limestone solubility in vitro, and calcium intake level on layer bone status and performance. Poultry Science, 69: 2214-2219.

Ccoicca, N. 2009. Efecto de la utilización de carbonato de calcio en fino o granulado en dietas de gallinas ponedoras sobre el espesor de la cascara y el comportamiento productivo. (Tesis título de Ing. Zootecnista). Universidad Nacional Agraria La Molina, Lima-Perú.
Cuca, M.; Valdes, V.; Pro, A.; Suarez, M. y Figueroa, J. 2007. Nivel de calcio y relación carbonato de calcio pulverizado: granulado y su efecto en producción de huevo y calidad del cascaron en gallinas ponedoras. Cusco, Perú: APPA-ALPA.

De Blas, C. y Gonzáles, M. 1991. Nutrición y Alimentación de Gallinas Ponedoras. Barcelona-España: Ed. Aedos.

Frost, T.; Roland, A. y Untawale, G. 1990. Influence of vitamin $\mathrm{d} 3,1$ alphahydroxyvitamin $\mathrm{d} 3$, and 1,25-dihydroxyvitamin $\mathrm{d} 3$ on egg shell quality, tibia strength, and various production parameters in commercial laying hens. Poultry Science, 69, 2008-2016.

Guinotte, F. y Nys, Y. 1991. Effects of particle size and origin of calcium sources on egg shell quality and bone mineralization in egg laying hens. Poultry Science, 70: 583-592.

HY-LINE Variedad Brown. 2011. Guía de Manejo Comercial. USA.

Keshavarz, K. 1994. Laying hens respond differently to high dietary level of phosphorus requirements in monobasic calcium phosphate. Poultry Science, 73: 687703.

Keshavarz, K. y Nakajima, S. 1993. Re-evaluation of calcium and phosphorus requirements, of laying hens for optimum performance and egg shell quality. Poultry Science, 72, 144-153.

Rao, S.; Roland, A. Y Gordon, R. 1995. Method to determine and factors that influence in vivo solubilization of phosphates in commercial leghorn hens. Poultry Science, 74: 1644-1649.

Rostagno, H.; Albino, J.; Doncele, P. y Oliveira, R. 2005. Tabelas brasileiras para aves e suinos. UFV. Departamento de Zootecnia, Vicosa MG.

Saunders, J.; Maclsaac, J.; Kover, D. y Anderson, D. 2009. The effect of calcium source and particle size on the production performance and bone quality of laying hens. Poultry Science, 88: 338-353.

Sauveur, B. 1993. El huevo para consumo. Madrid, España: Editorial Mundi Prensa.

Scott, M.; Neishem, M. y Young, R. 1991. Nutrition Of The Chicken. New York, USA: M. L. Scott and Associates. Ithaca.

Snow, J.; Douglas, M. y Parsons, C. 2004. Minimum requirement of one-cycle and two -cycle (molted) hens. Poultry Science, 83: 917-924.

Tobin, P. 2004. Influence of limestone particle size in layer diets on characteristic at peak production. (Thesis for to opt the degree magister scienteae agriculture). University of the Free State.

Williams, W. 2004. IV Seminario Internacional de Postura Comercial. Lima, Perú: Asociación Peruana de Avicultura. 